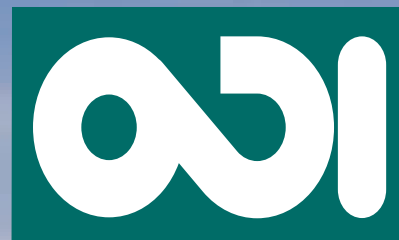

\title{
Measuring 'subjective resilience': using peoples' perceptions to quantify household resilience
}

Lindsey Jones and Thomas Tanner

Working paper 423

July 2015

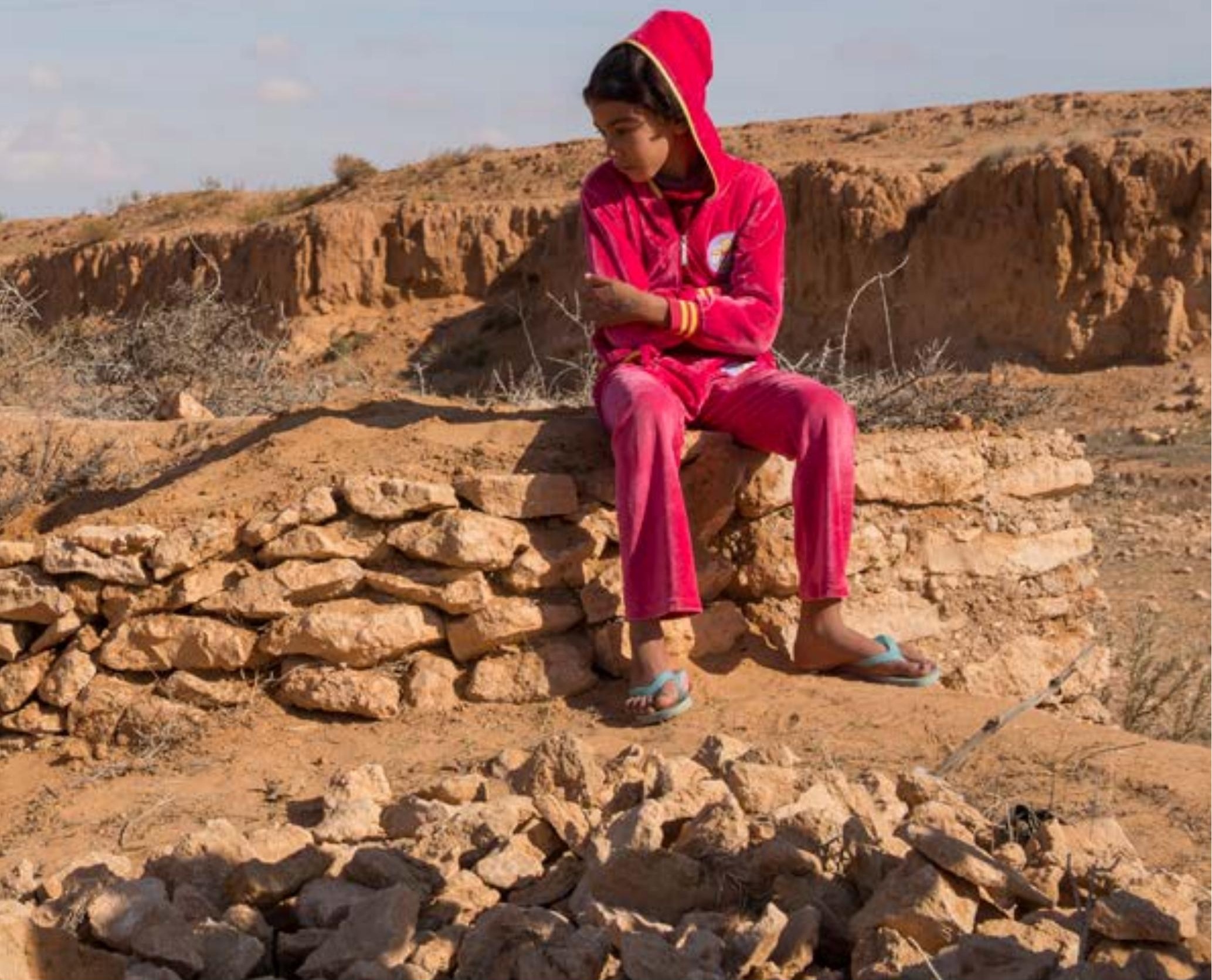


Overseas Development Institute

203 Blackfriars Road

London SE1 8NJ

Tel. $+44(0) 2079220300$

Fax. +44 (0) 2079220399

E-mail: info@odi.org.uk

www.odi.org

www.odi.org/facebook

www.odi.org/twitter

Readers are encouraged to reproduce material from ODI Reports for their own publications, as long as they are not being sold commercially. As copyright holder, ODI requests due acknowledgement and a copy of the publication. For online use, we ask readers to link to the original resource on the ODI website. The views presented in this paper are those of the author(s) and do not necessarily represent the views of ODI.

(C) Overseas Development Institute 2015. This work is licensed under a Creative Commons Attribution-NonCommercial Licence (CC BY-NC 3.0).

ISSN: 2052-7209

Cover photo: CGIAR Research Programme on Dryland Systems: Indigenous knowledge for resilience in marginal drylands - Tunisia 


\section{Contents}

Acknowledgements

Executive summary

1 Introduction

2 Understanding resilience

3 Rationale and foundation of a subjective approach

4 What can we learn from the use and application of subjective indicators in related disciplines?

5 What are the advantages of a subjective resilience approach to measurement?

6 What would an assessment of subjective resilience look like?

7 Challenges to the assessment of subjective resilience and how to address them

8 How can subjective resilience be used to inform decision making and better targeting of resilience-building activities?

9 Conclusion

References 


\section{List of tables, figures and boxes}

\section{Tables}

Table 1: Factors thought to influence the likelihood of error, response biases and heuristics

\section{Boxes}

Box 1: Examples that demonstrate the strengths and weaknesses of using a single question to evaluate subjective disaster resilience

Box 2: Examples of a set of questions used to evaluate subjective resilience 


\section{Acknowledgements}

The authors are indebted to helpful reviews and comments from Hallie Eakin and Emma Samman.

This paper has been made possible through the generous support of the Rockefeller Foundation. 


\section{Executive summary}

How should we measure a household's resilience? As resilience gathers momentum on the international stage, interest in this question continues to grow. So far, efforts to measure resilience have largely focused on the use of 'objective' frameworks and methods of indicator selection. These typically depend on a range of observable socioeconomic variables, such as levels of income, the extent of a household's social capital or its access to social safety nets. Yet, while objective methods have their uses, they suffer from well-documented weaknesses. Biases, such as the choice of indicators, the context-specific nature of resilience and the difficulties of capturing the less tangible processes that contribute to a person's resilience, all make the task of measurement difficult. Alternative approaches are therefore sought.

This paper advocates for the use of one such alternative: the measurement of 'subjective' resilience at the household level. The concept of subjective resilience stems from the premise that people have a good understanding of the factors that contribute to their ability to anticipate, buffer and adapt to disturbance and change. Subjective household resilience, therefore, relates to an individual's cognitive and affective self-evaluation of their household's capabilities and capacities in responding to risk.

In this paper, we discuss the advantages of measuring subjective household resilience. A subjective approach challenges the notion that experts are best placed to evaluate other people's livelihoods. It relies on people to self-assess and consider what characteristics are most important to the resilience of their household, providing a valuable opportunity to capture the perspectives of those who may know most about their own resilience: the people themselves. Thus, in some ways, the assessment of subjective resilience is more of a bottom-up process than traditional forms of 'objective' resilience measurement.

Resilience is heavily shaped by sociocultural and psychological factors such as risk perception, cognitive barriers and personal or cultural values. Given that the point of view rests with the individual directly, subjective forms of measurement allow for many of these 'softer' aspects of resilience - often difficult to capture through objective means - to be better factored in. Subjective approaches might also allow insights to be gained in contexts where accurate and large socioeconomic datasets are inadequate. While traditional forms of resilience measurement typically require collection and analysis of data across a large number of variables, subjective approaches are often shorter and direct: asking people to factor in all the aspects that contribute to their overall resilience, to rate their relative importance, and rate themselves accordingly. With this in mind, subjective approaches may lend themselves to the application of innovative forms of data collection. In particular, the rapid spread of mobile telephony throughout the developing world offers new means of subjective data collection.

A range of methods, surveying tools and applications can be used to measure subjective household resilience, each with their own methodological advantages and challenges. We therefore put forward different options for the design and delivery of survey questions on subjective resilience at the household level. While qualitative examinations of people's perceived resilience using open and semi-structured interviews may allow for in depth understandings, it is likely that the delivery of closedended questions will provide the most practical means of evaluating and comparing levels of resilience. The advantages of such approaches are that surveys can be administered quickly, are easier to code and interpret, and standardised. Importantly, they are also more readily quantified and may be comparable across spatial and geographic contexts. Subjective questions relating to resilience can either be asked as a single standalone question, or as a set of questions that probe different facets of a household's resilience: the former is simpler and easier to calculate, the latter is more complex to construct but far more holistic.

Care should be taken in examining the merits and limitations of various different approaches. Establishing the feasibility and methodological robustness of a subjective approach to measuring resilience will inevitably take time. However, a tremendous amount of knowledge can already be drawn from current understandings of household resilience. Insights can also be gained through the generation and use of subjective information in related fields, including perceived adaptive capacity, subjective wellbeing, and psychological resilience. While it is clear that any approach to subjective assessment will face significant methodological and conceptual challenges, we show that many are far from insurmountable.

Finally, we highlight how subjective household resilience can be used to improve policy and decisionmaking, through the evaluation and targeting of resilience-building activities, national and international resilience measurement, and the inclusion of bottom-up perspectives in decision-making processes at various levels of governance.

Ultimately, the aim here is not to entirely replace traditional methods of resilience measurement. Rather, if shown to be effective, we argue that bottom-up subjective methods should be used alongside objective methods, helping to capture many of the components of resilience that are difficult to observe and allowing people's perspectives to be heard in a systematic manner. Getting the process right will be an important step forward in gaining a more holistic understanding of what it takes for a household to be resilient to different forms of risk and how that resilience can be measured. 


\section{Introduction}

Resilience has rapidly risen to the top of the development agenda (Burnard, 2011; Frankenberger, 2014; Béné et al., 2013), and is now seen as a valuable conceptual tool in furthering the understanding of how people respond and adapt to the many changing shocks and stresses that affect livelihood outcomes (Manyena, 2006; Miller et al., 2010; Nelson et al., 2007). Inevitably, a push for resilience-building within the development and humanitarian communities has led to increased demand for ways of measuring levels of resilience amongst people and communities (Brooks et al., 2011). In theory, more accurate measurement and tracking of resilience can help to ensure that resilience-related policies and programmes are supporting the right activities and targeting the right people (Oddsdottir et al., 2013). Monitoring the effectiveness of resilience programming, rapid post-disaster assessment, and targeted social protection activities all involve the tracking of resilience on different scales (Frankenburger et al., 2014).

Unfortunately, the assessment of resilience is fraught with complexity: both the definition of resilience and the methodologies used to measure it are heavily contested (Cumming et al., 2005). Confounding factors, such as what mix of indicators to choose, which systems and scale of analysis to apply, and how to recognise the context-specific nature of resilience each muddy the waters. Indeed, despite growing global interest in supporting resilience-building activities, existing approaches to the measurement and tracking of resilience have generally not been able to the deliver the desired policy support (Levine, 2014).

A large number of frameworks and approaches have been proposed for quantifying household resilience (Bahadur et al., 2015). Most concentrate on 'objective' indicators by identifying key socio-economic variables and other capitals that support people's livelihoods. The selection of these variables is often value-laden and contested (Carpenter et al., 2001; Bahadur et al., 2015). They also typically require large and robust socioeconomic datasets, which are not easily found in many developing country contexts. However, a complementary means of assessing household resilience that can add value to and complement objective forms of measurement has largely been overlooked: 'subjective' household resilience. Subjective resilience stems from the premise that people have a good understanding of their own capacities, capabilities and limits. In leveraging more bottom-up processes of data collection, 'subjective' indicators of perceived resilience are based on behaviours, attitudes and psychology: factors not easily captured by traditional 'objective' indicators. The measurement of perceived resilience is therefore about how people rate their own resilience, and the resilience of the wider community of which they form part.

In this paper, we call for the tracking and measurement of subjective resilience at the household level. We describe why new approaches to measuring resilience are needed, what can be learned from other disciplines that use subjective indicators, and how subjective resilience can be adequately incorporated into methods of measuring and tracking resilience on the ground. We argue that efforts to measure resilience need to take people's perceptions of their own capabilities and capacities into account, either in combination with, or separate to, objective forms of resilience measurements. In order to narrow the context, we have chosen disaster resilience as the entry point for this paper, specifically the resilience of households to weather and climate extremes. However, the same principles would apply equally to other aspects of resilience, such as livelihood, community or social resilience, all of which possess many of the same characteristics.

\section{Understanding resilience}

As a concept, resilience has a wide variety of meanings and definitions. Although references to resilience can be found in art, literature, law, psychology and engineering (Alexander, 2013), the use of the term within the ecological sciences has been particularly influential, where 'resilience' is used to understand and explain the different trajectories of ecological systems as they seek equilibrium (Walker et al., 1969; Odum, 1985; Alexander, 2013). Ecological conceptualisations of resilience largely focus on the capacity of a system to absorb changes but still maintain its core function (Nguyen \& James, 2013). Holling (1973:14) describes resilience as 'a measure of the persistence of systems and of their ability to absorb change and disturbance and still maintain the same relationships between populations or state variables.' The term has been widely adopted as a way of framing the complex dynamics between linked social-ecological systems and their ability to respond to disturbance (Carpenter et al., 2001; Folke et al., 2002). Seen through this lens, social or livelihood resilience is used to assess the capacity of people and communities to prepare for and withstand shocks and stresses from a range of different hazards, whether environmental, social or economic (Eakin, 2012; Tanner et al., 2015).

These disciplinary transitions have also challenged traditional framings of resilience, namely that systems may not necessarily return back to the same function or existence after a perturbation (Olsson et al., 2015). More recent conceptualisations of resilience - mostly with regard to human systems - give greater recognition to the potential need of a system to adapt and change its core structures and functions, with some cases even requiring complete transformation (Pelling, 2003; Aldunce et al., 2015). Therefore, the resilience of a human system can be thought to comprise a range of different capacities and components, including, but by no means limited to: the capacity to absorb change (Nelson et al., 2007); preparedness and contingency (Twigg, 2009); innovation and learning (Adger, 2000); and renewal, reorganization 
and development (Folke, 2006). Knowing which components constitute the resilience of a system largely depends on the threat(s) (resilience to what?), the unit of analysis (whose resilience?) and the context.

Resilience's definitional and conceptual evolution has in turn made it difficult to agree on what constitutes a resilient human system. The abstract and malleable nature of the term, the lack of conceptual clarity, and strong overlaps with related concepts each make the process of conceptualising resilience difficult (Aldunce et al., 2015; Nelson et al., 2007). With regard to the latter issue, uncertainty about the relationship between resilience and similar properties such as adaptive, coping and transformative capacities remains a strong source of contention. Indeed, the terms are often referred to interchangeably across the academic literature (Bahadur et al., 2015). This is the case even for specific sub-fields of resilience:

In disaster management, [resilience] refers to multiple aspects ranging from absorbing and recovering from, to resisting, the effects of a hazard, as well as preserving and restoring "essential basic structures and functions". Such wide meanings may end up being contradictory as in the notion of "restoring equilibrium and getting away from it by moving to a new system state.

(Olsson et al., 2015:22)

A lack of clarity in how to apply resilience in practical terms and no clear consensus on what should and should not constitute resilience further muddy the waters, particularly when it comes to designing tools for the measurement of resilience (Djalante and Thomalla, 2011). Despite these challenges, the development and humanitarian communities have shown great interest in using the term to help guide operational activities and create greater cross-disciplinary linkages (DFID, 2014). This is evidenced by the growing quantities of international finance allocated towards 'resilience-building activities' (Peduzzi et al., 2009), including the Rockefeller Foundation's Asian Cities Climate Change Resilience Network (ACCCRN), the DFID-funded BRACED programme, and The Global Resilience Partnership, funded jointly by USAID, SIDA and the Rockefeller Foundation. With increasingly ambitious commitments and large international programmes dedicated to resilience building, there is a clear need to measure impact and track resilience on the ground. In spite of the conceptual ambiguities and inconsistencies, the race is on to determine the best ways of measuring resilience.

\section{Rationale and foundation of a subjective approach}

Similar to definitions of resilience, many different approaches, methods and frameworks for measuring resilience at the household level exist (Constas \& Barrett, 2013; FAO, 2009; Twigg, 2009; Elasha et al., 2005; USAID, 2012). In spite of such diversity, many quantitative measurement frameworks follow the same core steps, though not all approaches tackle them in the same way (Bahadur et al., 2015). The first step is to identify suitable characteristics of resilience through the observation of a particular system and drawing on the wider literature. Relevant 'objective' indicators are then assigned as proxies for each characteristic, typically drawing on the available socio-economic data. Lastly, in the case of single-item measures, these characteristics and indicators are amalgamated into a composite index, often with indicators being weighted differently.

While such approaches have operational benefits, they are not without weaknesses. For one, it is extremely difficult to identify all the relevant traits and indicators from economic to socio-cultural and political factors - that influence a household or community's resilience (Cutter et al., 2008). Approximations have to be made, and this places considerable weight on the choice of framework and characteristics used. The context-specific (and scale-specific) nature of resilience also means that identifying the right indicators is challenging: what contributes to resilience in one community may not have the same effect in another (Engle, 2011). Measures determined from the top down may favour more structural determinants at the expense of those based on human agency, which may be harder to understand and measure (Tanner et al., 2015). In addition, the range of different data sources and inputs needed in compiling such indices means that large household surveys are usually required, which are often costly and timeconsuming. Crucially, this top-down approach speaks little to how people evaluate their own lives, and often requires value judgements to simplify the complex nature of resilience across so many different contexts (Diener et al., 2002). Such value judgements mean that these measures of resilience are often far from 'objective'.

However, there are alternatives that offer significant advantages in terms of complementing traditional approaches to resilience measurement. 'Subjective' resilience is one such alternative.

Subjective resilience can relate to two important (and overlapping) factors. Firstly, it relates to the notion that a person's resilience is comprised not only of tangible objective elements, such as the availability of various livelihood assets, but also wider social, cultural and psychological elements (Adger et al., 2013). The subjective elements of resilience are associated with a range of issues such as perception of risk, sense of place, beliefs and culture, social norms, social cohesion, power and 
marginalisation, and cultural identity (Grothmann and Patt, 2005; Adger et al., 2009; Clayton et al,. 2015). Despite the difficulty of assessing many of these factors, they are nonetheless crucial to household and community resilience. Current assessments and conceptualisations of resilience seldom capture these more subjective elements (Brown \& Westaway, 2011). Factoring them into evaluative frameworks is therefore key to gaining a more holistic understanding of resilience, particularly at the household and community levels.

The second factor relates to the subjective assessment of an individual's own resilience or the resilience of others around them, whether at the personal, household or community level (Marshall, 2010). This topic has been far less studied and is therefore the primary focus of this paper. We define subjective resilience in terms of people's perceived level of household resilience to specific external shocks and stresses. It relates to a person's cognitive and affective valuation of their own capacity to anticipate, buffer and adapt their livelihoods to disturbance and change.

The relationship between these two factors of subjective resilience is complex. On the one hand, psychological and cultural elements will inevitably affect how a person rates their household's ability to respond to disasters. For example, two members of the same household - perhaps one with the personality traits associated with overt optimism, the other with pessimism - may well rate their household very differently. Thus, in many ways subjective elements can act as a significant bias to subjective selfassessments of a household's capacities. On the other hand, these same psychological and cultural elements also have a profound influence on household resilience. For example, cultural norms such as ethnic marginalisation will impact the ability of certain social groups to respond to disasters, perhaps through restricted access to key resources or economic marginalisation (Burton \& Cutter, 2008). Individual subjective traits, such as risk aversion or risktaking, may also affect how a household chooses to respond to disaster risk and therefore influence their household's overall resilience. Any self-assessment of household capacities, therefore, has to be mindful of the distinctions between these two potentially opposing traits, and seek ways of recognising and accounting for relevant biases.

If care is taken to design suitable methodologies for data sampling and collection, then a household's subjective resilience can, in theory, be readily quantified and used as a complementary approach to objective resilience measurement. Importantly, assessments of subjective resilience are also subject to response bias, affected by context and difficult to translate into different languages. However, they offer the opportunity to complement and significantly enhance current resilience measurement practices.

The concept of subjectively defined resilience draws many parallels with the paradigm shift that arose from subjective approaches to the measurement of well-being, which was traditionally measured solely through objective approaches (Brown \& Westaway, 2011). Indeed, the role of 'subjective' indicators is increasingly being recognised by other overlapping subject areas such as well-being, food insecurity and poverty. This recognises the advantage of being able to better capture the multi-faceted nature of these concepts and allows for data generation from the bottom up. Such practices have yet to trickle down to resilience measurement methods, but their potential contribution is outlined in the next section.

\section{What can we learn from the use and application of subjective indicators in related disciplines?}

Subjective resilience has strong overlaps with related subfields across the social sciences. Below we highlight three in particular: perceived adaptive capacity, well-being, and psychological resilience. We briefly outline close conceptual similarities amongst the approaches, potential influences on subjective resilience, as well as opportunities to explore novel methods for resilience assessment.

\subsection{Perceived adaptive capacity and social resilience}

A number of studies within the literature on climate change adaptation have explored aspects related to subjective disaster resilience at the household level (Adger et al., 2009; Brown \& Westaway 2011; O’Brien, 2009: Nguyen and James, 2013). Most have sought to develop conceptual frameworks to better understand the factors that contribute to risk perception, individual capabilities and capabilities. For example, Grothmann and Patt (2005) discuss the idea of 'perceived adaptive capacity' and explore qualitative case studies of proactive adaptation in Germany and Zimbabwe. They theorise that perceived adaptive capacity is comprised of three sub-components: perceived adaptation efficacy, perceived self-efficacy and perceived adaptation costs. Frank et al. (2010) expand on this using the case of coffee farmers in Chiapas, Mexico: they posit that social identity is an inherent additional component of an individual's perceived risk and adaptive capacity.

The majority of such studies remain heavily guided by the qualitative assessment of targeted case studies. This is perhaps unsurprising given the strong influence of social, psychological and institutional factors. Few have attempted to quantify these relationships. Where this has been done, questions have been raised as to the suitability of identified components. For example, Blennow et al. (2012) and Blennow \& Persson (2009) investigate the factors that contribute to perceived adaptive capacity in the Swedish forestry sector. They find little evidence to support Gothmann and Patt's (2005) three sub-components, instead showing strong links between adaptive capacity and two further characteristics: personal belief in climate change and perception among those who make decisions for adaptation at the local level. 
Other relevant examples include a survey of 100 commercial fishers carried out by Marshall and Marshall (2007) to investigate levels of 'social resilience' in five coastal communities in North Queensland. By asking 17 questions related to the characteristics of resilience (later reduced to 12), responses to generic yet anticipated change events were explained by four characteristics: perception of risk associated with change; perception of the ability to plan, learn, and reorganise; perception of the ability to cope; and level of interest in change. The same methods were adopted and trialled, again in the context of fisher communities, through 157 surveys in ports across Rhode Island, Massachusetts and the Cape Cod region of Massachusetts, linking well-being, resilience and job satisfaction (Seara, 2014). A similar approach was also investigated by Marshall (2010) among cattle grazers in Australia; it highlighted that the highest resilience was associated with grazers who used seasonal climate forecasts, were highly attached to 'place', employable, strategic and financially secure. In attempting to use a psychometric approach, Lockwood et al. (2015) explore dimensions of 'adaptive capacity and personal resilience', indicating that the most important factors influencing perceived landholder adaptive capacity amongst rural landowners in South-Eastern Australia are related to landholders' change orientation, financial capacity and the ability of communities to support individual landowners.

While such approaches have paved the way for a better understanding of the characteristics and components of perceived adaptive capacity and social resilience, few have provided the details of a systematic process of identifying base questions, with the exception of Lockwood et al. (2015). Fewer still have taken the next step and sought to use subjective aspects of resilience to help guide resilience-building initiatives and policies (Marshall, 2010). In addition, the case study focus of most existing research efforts limits the scope for wider conclusions and applications to be drawn. Exploring the development of a standardised set of queries that can be used to assess subjective disaster resilience in any given context would be key going forward. Not only will this require considerable further research and ground-truthing, but also the acknowledgement that certain contextual factors may be lost in exchange for scalability, replicability and comparability (applications for standardised subjective resilience approaches are further explored in the sections below). In many ways, this is similar to the approaches adopted by many national and global polling exercises in the measurement of subjective well-being.

\subsection{Subjective Well-being}

Perhaps the field most closely related to subjective resilience is well-being. Buoyed by the recognition that a country's progress and development should be measured not just by its GDP but wider measures of economic, social and environmental impact (Costanza, 2009), the assessment of well-being has received considerable attention both from the research and policy communities (Diener, 2000; OECD, 2013). A wide number of studies and renewed interest in the concept reveal that the concept of well-being is truly multi-faceted:

'Well-being can be understood as how people feel and how they function, both on a personal and a social level, and how they evaluate their lives as a whole. To break this down, how people feel refers to emotions such as happiness or anxiety. How people function refers to things such as their sense of competence or their sense of being connected to those around them. How people evaluate their life as a whole is captured in their satisfaction with their lives, or how they rate their lives in comparison with the best possible life.' (NEF, 2012)

Well-being is commonly measured in two ways: either through objective or subjective indicators. Objective well-being (OWB) operates from the point of view that a person's well-being is determined by the extent to which they satisfy a predefined list of requirements deemed to contribute to a 'good life' (Guillen-Royo \& Velazco, 2006; Valerius, 2004). This list typically includes entries such as income, access to healthcare, education, social capital, and food consumption. These requirements are usually universal and do not vary among different social groups (Guillen-Royo \& Velazco, 2006).

Subjective well-being (SWB) operates from the point of view of the individual, arguing that people are 'the best judges of the overall quality of their lives, and it is a straightforward strategy to ask them about their wellbeing' (Frey and Sutzter, 2002, 405). SWB can be thought of as people's multidimensional evaluation of their own lives, including cognitive judgments of life satisfaction as well as affective evaluations of moods and emotions (McGillivray \& Clarke, 2006). In aiming to capture SWB, researchers typically rely on self-assessments of life satisfaction and well-being. Neurological research also lends support to the view that life satisfaction measures are related to individuals' emotional states (Kahneman \& Krueger, 2006) and correlated with the left-right different in brain activation (Urry et al., 2004).

The relationship between objective and subjective wellbeing is complex. While weak relationships exist between income and SWB, these are not uniform. Economic growth in developed countries has not been associated with a rise in SWB in the past decades, seemingly stopping beyond middle-income countries (known as the Easterlin Paradox) (Guillen-Royo \& Velazco, 2006). However, more recent assessments present a challenge to this hypothesis, suggesting that GDP is positively linked with GDP per capita across countries (Stevensen \& Wolfers, 2008). Other discrepancies exist between OWB and SWB directly. For example, in welfare economics, Sen documented the case of women in India, who had considerably worse 
objective health status than comparable men, yet declared themselves more subjectively content with their situation than men (Sen, 1985; Gapter, 2005).

The discrepancies between subjective and objective measures of well-being make a strong case for the investigation of similar properties in the context of resilience. For the moment, objective measures of resilience are the predominant instrument used. Given that it is likely that perceptions, norms and behaviours play a strong role in shaping a household's resilience (McIvor \& Paton, 2007; IFRC, 2014), an understanding of the relationship between objective and subjective resilience will undoubtedly add considerable value to this emerging field of research. In addition, the relative success with which SWB has been able to galvanise policy interest around the importance of moving past objective measures of economic and social well-being, both internationally and nationally, may have strong parallels to the resilience agenda.

\subsection{Psychological resilience and disaster psychology}

The entry-point for much of the work on psychological resilience is the individual psyche. It seeks to understand the ability of individuals to cope with and adapt positively in the face of loss, hardship or adversity (Singh \& Yu, 2010). This research has examined a wide range of determinants of personal resilience including epigenetic, developmental, psychosocial, and neurochemical factors (Wu et al., 2013). Others have sought to situate these individual responses within the wider contexts of social and physical ecologies that link individual risk, social organisation and culture (Ungar, 2011).

Four waves of research on psychological resilience are determined by Masten (2011) to have focused on (1) defining the meaning of resilience across contexts and disciplines; (2) how to promote resilience; (3) modelling resilience processes; and (4) measuring resilience, especially through the use of statistical analysis to understand mediators and moderators of risk and resilience.

Many different methods of measuring psychological resilience exist. Several of these focus on clinically robust quantitative methods, including longitudinal cohort studies, cross-sectional thematic qualitative studies, and randomised control trials (Graber et al., 2015). In their systematic review, Windle et al. (2011) identify 15 such measures of psychological resilience. While there is considerable methodological diversity, most approaches fall under two categories. The first uses a person's self-evaluation of prior experiences of successfully overcoming stressful events and positive changes. This method requires a particular stress event to occur, or a person's recollection of their response to a previous one. The second measures subjective factors deemed to be determinants of resilience, such as personal competence or social resources. This approach 'may prospectively determine resilience but does not evaluate resilience itself' (Scali et al., 2012:1). Both are relevant and applicable in the context of subjective resilience.
Of the former category, one of the most commonly applied and studied is the Connor Davidson Resilience Scale (CD-RISC). CD-RISC is a self-administered scale of 25 questions testing psychometric properties that cover five factors corresponding to: personal competence, high standards, and tenacity; trust in one's instincts, tolerance of negative affect, and strengthening effects of stress; positive acceptance of change and secure relationships with others; control; and spiritual influences (Singh \& Yu, 2010). A shortened version featuring 10 questions has also shown to be as effective, and concentrates on the ability of person to bounce back from the variety of challenges that can arise in life (Scali et al., 2012).

Similar systematic approaches to the selection of questions for a subjective resilience scale may also prove to be useful given the overlap between psychological and subjective resilience. Indeed, a person's psychological resilience will inevitably have a strong influence on how resilient they perceive their household or community to be. Factoring this into any assessment of subjective resilience will therefore be key, particularly in acknowledging and correcting for any biases. Other methodological challenges familiar to psychological resilience research include the non-linear nature of resilience development, how to mix subjective and objective indicators, the use of informant reports, difficulties recording baseline or pre-trauma functioning, confusion between resilience and associated constructs such as self-esteem, and the need for clarity between resilience outcomes and resilience processes (Graber et al., 2015).

\section{What are the advantages of a subjective resilience approach to measurement?}

There are many reasons why subjective disaster resilience can add value to objective methods of measurement. First, it takes advantage of the fact that people have a good understanding of their capabilities and capacities to deal with disturbance and change (Nguyen \& James, 2013). They are also aware of many of the factors that enable or constrain the resilience of their livelihoods (Marshall et al., 2010). By defining objective indicators, it is assumed that external actors - typically in the form of resilience 'experts' - know what these factors are, and set the parameters accordingly to predefined frameworks. A subjective approach to measurement challenges the notion that experts are best placed to evaluate other people's lives, and have a better understanding of the factors that contribute to people's own resilience (Diener et al., 2002). Thus, in some ways, the assessment of subjective resilience is more of a bottom-up process. It relies on people to self-assess and consider what characteristics are most important to their own livelihoods. While agent-based assessments are not without weakness or bias (see Table 1), they offer valuable insights that should be considered alongside traditional objective measures of resilience. 
Second, subjective measures of resilience can help to reduce uncertainty in the selection of indicators. The range of indicators used under different methods for measuring objective resilience is vast. The Sustainable Livelihood Framework's five capitals (Elasha et al., 2005; Lockwood et al., 2015; Uy et al., 2011; Keating et al., 2014), variants of the Human Development Index (Briguglio et al., 2007), and many other individual frameworks (Speranza et al., 2014; Pasteur, 2011; Fox et al., 2012) each have their own set of indicators used to characterise livelihood resilience. Subjective measures can get around this somewhat, as questions are often direct: asking people to factor in all the aspects that contribute to their overall resilience, to rate their relative importance, and rate themselves accordingly. This can often be done through asking a small number of considered questions (examples of the types of questions that could be used are explored in Sections $6 \& 7$ ). In this way, it navigates the need for dozens (in some cases hundreds) of different objective indicators, and the equally subjective choice of which ones to include or exclude. Assessments of subjective resilience do not avoid the challenge of selection and bias entirely however, as the choice and wording of questions will inevitably require judgment calls, as will the selection of what (and how many) questions to ask (see Sections 5 \& 6).

Third, it allows insights to be gained on resilience in contexts where accurate and large socioeconomic datasets are inadequate. Measurement of objective resilience typically requires the collection and analysis of data across a large number of variables. Subjective assessments in other related fields are typically done using a limited number of questions that are further reduced using statistical techniques. For example, in the case of subjective ratings of health status, the widely used RAND short form health survey is a set of 36 generic, coherent, and easily administered quality-of-life measures (Hays et al., 2006). The same survey is further reduced to even shorter 12 and 6 questions which have proven effective in capturing much of the detail of the full survey (Bowling 2005). It may well be possible that a subjective assessment of resilience can lend itself to a similar process of identifying a subset of suitable questions that capture the detail and variance of a larger survey. With this in mind, such an approach may lend itself readily to the application of more innovative forms of data collection, such as crowd sourcing through ICTs. In particular, the rapid spread of mobile telephony throughout the developing world offers new means of data collection. Penetration in Africa is rising exponentially (hitting $80 \%$ in 2013), allowing information to be harnessed in difficult to access places in a near-real-time manner. SMS and voice-based surveys can be administered at a fraction of the cost of traditional household surveys, and allows the leveraging of 'Big Data' to more readily track and measure resilience. Indeed, such techniques open up novel opportunities such as the ability to: track resilience during or immediately after an identified shock (such as a flood event or heat-wave); quickly administer pre $\&$ post evaluations of resilience-building interventions amongst recipients; and easily collect large-scale longitudinal data. In taking advantage of these qualities, it is likely that such approaches will allow for data collection in areas difficult to access through traditional household survey teams, such as in fragile and conflict-affected areas.

Lastly, resilience is heavily shaped by sociocultural and psychological factors such as risk perception, cognitive barriers and personal or cultural values, which can each play a key role in determining whether adaptation is sought, or whether people have access to vital resources in times of need (Kuruppu and Willie, 2014; Jones and Boyd, 2011). Given that the point of view rests with the individual directly, subjective indicators allow for many of these 'softer' aspects of resilience - often difficult to capture through objective means - to be better factored in. In turn, this also brings limitations with it, as cultural factors can present an inherent bias to self-reported score. Subjective household resilience would face similar challenges of having to account for 'cultural measurement bias' and the effects of emotions and norms as seen in the measurement of subjective well-being (Suh, 1998). For example, in collectivistic societies, such as Japan, people will tend to present themselves as 'average' citizens, scoring themselves as less happy than they are (Iijima, 1982). Could it also be the case that resilience is culturally relative? Though these present significant methodological obstacles, they have shown not to be limiting factors, and do not lead to significant cross-national differences in scores in other related fields such as subjective well-being and happiness (Veenhoven, 2012; Veenhoven, 1990).

\section{What would an assessment of subjective resilience look like?}

What would a question, or set of questions relating to subjective resilience look like in practice? Although the process of asking people questions about their perceived levels of resilience may at first seem straightforward, it is anything but. There is a multitude of ways of asking questions relating to subjective resilience, each with its own methodological challenges and biases. Careful thought therefore needs to be placed in designing and delivering questions to ensure the robustness and utility of subjective information.

To begin with, there are many different types of 'resilience' referred to in the literature. These include: personal resilience, psychological resilience, livelihood resilience, community resilience, social resilience, economic resilience and disaster resilience, to name but a few. While there are many overlaps between them, each is focused on the characteristics that make their respective systems resilient to particular threats. Each is also applied at a specific geographical scale and unit of analysis. Thus, the characteristics and properties of an individual's psychological resilience may not be the same as those that make up a 
Table 1: Factors thought to influence the likelihood of error, response biases and heuristics

\begin{tabular}{|c|c|}
\hline $\begin{array}{l}\text { Factors associated with the underlying } \\
\text { construct of interest }\end{array}$ & Survey design factors \\
\hline $\begin{array}{l}\text { Task difficulty } \\
\text { How easy or difficult is it for respondents to think } \\
\text { about the construct or recall it from memory? }\end{array}$ & $\begin{array}{l}\text { Question wording } \\
\text { Is the wording complex or ambiguous? Can it be } \\
\text { easily translated across languages and cultures? Is } \\
\text { the tone of the question sufficiently neutral, or does it } \\
\text { suggest particular answers should be favoured? }\end{array}$ \\
\hline $\begin{array}{l}\text { Translatability } \\
\text { How easy or difficult is it to translate the construct } \\
\text { into different languages? }\end{array}$ & $\begin{array}{l}\text { Response formats } \\
\text { Is the wording complex, ambiguous or difficult } \\
\text { to translate? Can the response options be easily } \\
\text { remembered? Can respondents reliably distinguish } \\
\text { between response categories? Are there enough } \\
\text { response categories to enable views to be expressed } \\
\text { fully? }\end{array}$ \\
\hline
\end{tabular}

Respondent factors

Motivation

Are respondents equally motivated?

Fatigue

Are respondents equally alert and engaged?

Susceptibility to social pressure, norms or demand characteristics

Do respondents vary in terms of their

susceptibility to social pressure/or their

likelihood of responding in a socially desirable manner?

\section{Language differences}

Do language differences between respondents

influence how respondents interpret questions and response formats?

associated with the construct, i.e. normatively

"good" and "bad" answers?

Do preceding questions influence how an item

is interpreted and/or prime the use of certain

information when responding?

Cultural differences

Do cultural differences affect the type of response biases or heuristics that might be seen when respondents are satisficing?*

(demand characteristics) or promote socially desirable responding?

\section{Survey mode}

Does the survey mode influence respondent

motivation, response burden (e.g. memory

burdens) and/or the likelihood of socially desirable

responding?

Respondent interest/engagement

How relevant or interesting do respondents find

the construct being measured?

\section{Knowledge}

Do some respondents lack the knowledge or experience to be able to answer the question (but attempt to do so anyway)?

Cognitive ability

Do respondents vary in their ability to

understand the question and/or in their

memory capacity?

\section{Wider survey context}

Does the day of the week or the time of year affect responses? Could day-to-day events (such as major news stories) or the weather influence responses?

\footnotetext{
* Satisficing is when a respondent answers a question using the most easily available information rather than trying to recall the concept that the question is intended to address. A satisficing respondent may make use of a simple heuristic to answer the question or draw on information that is readily available in their mind rather than trying to provide a balanced response.
}

Source: OECD, 2013

country's economic resilience. The first step in designing an assessment of subjective resilience is therefore to decide on the type and scale of resilience one wishes to investigate.

The example used in this paper to illustrate the potential for subjective assessments is a sub-set of disaster resilience. Specifically, we are interested in the resilience of households to respond to weather and climate-related extremes. We define this as the ability of households to manage change by maintaining or transforming living standards in the face of shocks related to weather or climate events - such as droughts, floods or the delayed onset of rainfall seasons - without compromising their long-term prospects (adapted from DFID, 2011). This focus on disaster resilience can either relate to a single hazard or an aggregate of multiple hazards. A subjective assessment of any of the different types of resilience listed above is entirely feasible, but though would require a different set of questions and wording.

The assessment of subjective resilience can be undertaken using many different evaluative survey techniques. Given the multifaceted nature of resilience, perhaps the most robust manner of collecting information is through open-ended questions, whereby a series of semi-structured (or structured) questions are administered, allowing people to freely reflect on how resilient they perceive their household or livelihood to be. This method 
allows for rich qualitative data to be collected without prescribing responses. However, open-ended questions and surveys are often difficult to quantify. They also require considerable human and technical resources in collecting relevant data at scale.

The most practical and useful means of collecting information on subjective resilience may therefore be through the delivery of structured surveys. Here, a fixed list of questions and answers that limit the respondents to pre-selected answers from which respondent are requested to choose are administered. The advantage of such an approach is that surveys can be administered quickly, are easier to code and interpret, and standardised. Most importantly, they are more readily quantified. Typically, this type of approach is accompanied by either dichotomous (two-point), multiple choice or scaled questions (such as those reliant on Likert scale responses).
However, they can also lend themselves to visual analogue scales or even be combined with open-ended responses.

Before delving into the specifics, it is first important to consider the options that exist in formulating a single close-ended question relating to subjective resilience. Small differences in the way a question is constructed can have large implications for respondent comprehension, reporting and the comparability of data collected (see Table 1). Questions that are easy to understand, low in ambiguity and do not burden the respondents should be sought (OECD, 2013). With the assessment of household resilience to weather and climate extremes in mind, one of the first challenges is to specify the threat that is being assessed. Two options exist: a question could either relate to the ability of households to respond to the impacts of a singular stressor, such as drought (see Q1 in Box 1); or it could relate to the collective impact of weather-related

\section{Box 1: Examples that demonstrate the strengths and weaknesses of using a single question to evaluate subjective disaster resilience}

[Q1] "All things considered, how resilient is your household to the threats posed by drought? Very resilient; somewhat resilient; or not at all resilient?"

Pros: Concise and simple question and response items; targets a specific hazard.

Cons: 'Resilience' is ambiguous and has many interpretations; using three response items substantially limits detail.

[Q2] "How resilient is your household to threats posed by extreme weather events? Using the scale below, on which 0 means 'not at all resilient' and 10 means it is 'very resilient', how resilient would you rate your household as a whole?

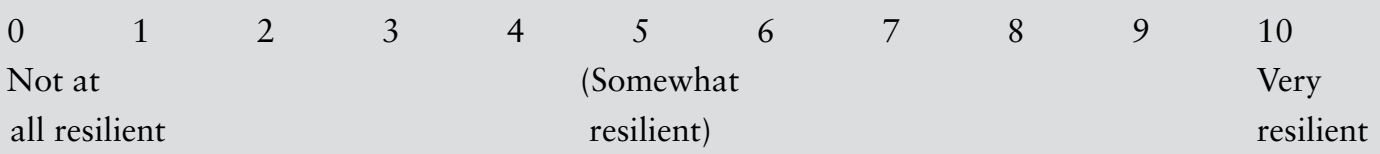

Pros: Short and concise question; covers a range of threats; comprehensive response item; visual aid.

Cons: 'Resilience' is ambiguous and has many interpretations; scale may be confusing to those unaccustomed to it; difficult to showcase verbally; heavy importance on correct labelling of response terms.

[Q3] "At this point in time, I consider my household to be resilient to threats posed by [insert a singular hazard or refer to term that aggregates multiple hazards]?” Agree; disagree

Pros: Reference period; binary response items leave little ambiguity.

Cons: 'Resilience' is ambiguous and has many interpretations; limiting response to two items means the degree of detail is restricted.

[Q4] "Compared with last year, my household is much better at coping with and adapting to the threats posed by extreme weather events?” Rated on a 7, 5 or 4-point scale from Strongly Disagree (1) to Strongly Agree (7).

Pros: Reference period; doesn't mention word 'resilience'; widely used Likert scale allows for depth in answers.

Cons: Ability to cope may be different to ability to adapt; points on the scale may be affected by understandings of each term.

[Q5] " If heavy flooding was to occur in my area tomorrow, my household would be able to successfully deal with the threats posed by the floods." Please use a scale from 0 to 10 to indicate how you feel with regards to the above statement. Zero means you "disagree completely" and 10 means "agree completely".

Pros: Reference period; wide ranging response items; encourages reflection.

Cons: Points on the scale may be affected by understandings of each term. 
extremes $(\mathrm{Q} 2)$ - this would imply the full range of weatherrelated extreme events that may affect that particular household, such as floods, droughts and more variable rainfall events. The former is specific, easier to comprehend and therefore likely to provide answers that are more robust and tailored to a particular threat. While the latter is more vague in its construction and prone to ambiguity - a household may be very resilient to flood events but not at all resilient to drought - its generalisability allows for it to be applied across a wider range of contexts and derive useful information in relation to the many weather-related threats that affect household disaster resilience. This is critical when considering resilience as a wider approach to securing development in the face of a range of shocks and stresses. Choosing between the two approaches is therefore dependent on the research aims and objectives. While there is no right or wrong approach, users should be aware of the merits and limitations of each.

A second, related challenge is deciding on the structure of the question. Precise wording is key, particularly when there are ambiguities with regards to definitions. For example, Q1 in Box 1 presents a simple and direct way of formulating a resilience-related question. However, the term 'resilience' means different things to different people. There may also be difficulties in translating it effectively across languages. One option, therefore, is to omit the word 'resilience' in the question and allude to its characteristics. For example, Q4 instead refers instead to the ability of a household to cope and adapt to climate extremes. However, it is very difficult to cover the multifaceted nature of resilience in a single question without sacrificing the validity and utility of the information gleaned from the question. In addition, any singular question that refers to two separate capabilities may elicit different responses and confuse respondents: i.e. referring to Q4, my ability to cope with increased flood risk may be different to my ability to completely adapt my livelihood in response to continued flood risk.

Another consideration is the time period of assessment. This is particularly relevant to resilience, as it is comprised of both short-term (e.g. absorptive/coping capacity) and long-term (adaptive capacity) components. Thus, it is important to make reference to the specific time period (and capacity) within the structuring of all relevant questions. For example, questions Q3, Q4 and Q5 each ask respondents to sum up their experiences over a given reference period - either in relation to the present time or in comparison with a stated period. Alternatively, leaving out reference to a specific time period will likely imply that respondents indicate their views at the present moment while drawing on their experiences from the close (and potentially distant) past.

Equally challenging is deciding on the format of response options. Question designers need to consider how many responses to offer, how to label them as well as the scale of intervals. More importantly, they have to decide

\section{Box 2: Examples of a set of questions used to evaluate subjective resilience}

A subset (or all) of the following items may be rated on a 7- or 5-point scale ranging from Strongly Disagree (1) to Strongly Agree (7):

[Q6] 'If heavy flooding was to occur in my area tomorrow, my household would be able to successfully cope with the threats posed by the floods' OR 'If heavy flooding was to occur in my area tomorrow, my household would be able to fully recover from the damage caused by the floods within 6 months.'

\section{Component of resilience: Coping capacity}

[Q7] 'If the rate and intensity of flooding was to increase significantly in the next 5 years, my household would have the ability to successfully adapt to the changing threats posed by the floods' OR 'If the rate and intensity of flooding was to significantly increase in the next 5 years, my household would have the ability to successfully adapt to the changing threats posed by the floods, even if this required us to completely change our way of life.'

Component of resilience: Adaptive capacity (the latter is explicitly probing transformative capacity)

[Q8] 'If heavy flooding was to occur in my area tomorrow, my household would have access to sufficient financial resources to ensure that we fully recover from the threats posed by the floods.'

Component of resilience: Financial capital

[Q9] 'If heavy flooding was to occur in my area tomorrow, my household would be able to draw on the support of family and friends to ensure that we fully recover from the threats posed by the floods.'

Component of resilience: Social capital

[Q10] 'My household has learned considerably from how we have dealt with past drought events. This knowledge is crucial in successfully dealing with future drought events.'

Component of resilience: Iterative learning

[Q11] 'If heavy flooding was to occur in my area tomorrow, my household would have access to early-warning information to ensure that we are fully prepared for the threats posed by the floods.'

Component of resilience: Knowledge and information

on whether questions regarding subjective resilience should be measured on a bipolar scale (e.g. agree/disagree) or a unipolar scale (e.g. not at all - completely), and whether respondents should be asked for a judgement involving frequency (how often do you feel...?) or intensity (how resilient do you feel...?) (OECD, 2013). Examples of 
different types of response items, and the various pro and cons associated with each are presented in Box 1.

As with many of the choices described above, each method of designing response options should be tailored to the needs of the user. Some may choose to prioritise concise and short responses (see Q1 and Q4) to limit ambiguity and make cross-country comparison or longitudinal analysis easier. Yet, this will reduce the level of detail that can be extracted from the answers (particularly in the case of binary answers) (Cummins, 2003). Note that in the context of subjective resilience, single question answers are likely to be unipolar (running from low resilience to high resilience) rather than bipolar (between two opposing constructs resilient/unresilient). Others may choose to allow for a greater number of response options to allow for such detail. However, increasing numbers beyond the optimal length can result in information loss, increased error and reduced motivation (ibid.). 5 and 7-point scales remain the most common options within the context of most life evaluation surveys, though there is an increasing number of surveys using higher point scales (typically 11-point). Choosing meaningful labels that are easy communicable, translatable and adequately reflect each of the gradients on the point scale is an equally important consideration.

Drawing on experiences from related fields, it is likely that questions administered to assess subjective resilience to weather-related extreme events (or any other types of resilience) would consist of two main delivery options. The first is to have a simple standalone single-item question (see Fordyce, 1988). This approach has long been used in assessments of SWB. Examples of stand-alone SWB questions include: "All things considered, how satisfied are you with your life as a whole these days?" or "Taken all together, would you say that you are very happy, pretty happy, or not too happy?" These questions aim to elicit an easily replicable global evaluation of one's life (Krueger \& Schkade, 2008). They also seek to be as universally applicable as possible in order for comparison (both with other geographic contexts and across time). A similar approach could no-doubt be adopted for the assessment of subjective disaster resilience. The aim being to design a question that could, to the best possible extent and recognising the limitations associated with it, give an accurate account of a person's perceived level of household resilience with a single question. With this in mind, each of the examples presented in Box 1 showcase the types of questions that could be applied as a single question to assess subjective disaster resilience (note that the design of each question is meant to highlight the strengths and weaknesses of different approaches).

The weaknesses in a single-question approach become quickly apparent. Primary amongst them is the difficulty in condensing the different components of resilience into a single concise question. To counter some of these methodological challenges, a second approach would be to ask a series of questions related to aspects known to affect disaster resilience (see Box 2). Each question would probe a different aspect of disaster resilience, aiming to provide a more holistic response. We would consider this to be a far more appropriate way of measuring subjective disaster resilience. For example, a similar approach is taken by the widely used Satisfaction with Life Scale (SWLS), identifying five related questions that are then used as global measure (Diener et al., 1985). Typically, these questions are then grouped or consolidated to form a composite index. A number of different statistical techniques (such as Principal Component Analysis or various regression-based approaches) can be applied to either identify a small set of questions from a larger subset (that account for much of the variance), or to assign relevant weightage to each question.

As with a single item question, multiple questions and composite indexes also have their methodological challenges. To begin with, agreeing on which (and how many) questions to include is inevitably difficult and subjective. Indeed, it is possible for numerous different combinations to arise. For example, in the case of psychological resilience for example, Windle et al. (2011) identify nineteen different methods of assessment in the academic literature, each with their own way of questioning, classifying and weighting within their respective resilience scales.

One approach would be to start with a clean slate and use bottom-up qualitative research to identify questions that people and communities themselves consider as best representing the characteristics of a resilient household indeed, questions identified under the first approach may be 'ground-truthed' by the latter. This would help avoid expert-led bias, but require extensive initial pilot surveying in order to develop the subset of question areas.

Another option would be to isolate particular characteristics of resilience, and assign a small number of questions that relate to each characteristic. These questions could be drawn from the wider literature and would then be grouped and weighted accordingly. For example, given that resilience is often broken down into three interrelated capacities (Folke, 2002) - the capacity to cope; the capacity to adapt; and the capacity to transform - questions could quite easily be identified to suit each. See Q5 and Q6 that probe different capacities associated with resilience. The five livelihood capitals (Scoones, 1998) are also closely associated with household resilience (Eakin \& Wehbe, 2009) and could be used as the basis for understanding and probing subjective assessments of resilience - see questions Q8 and Q9. In addition, resilience is often characterised as being comprised of various different processes and functions, such as the iterative learning, accessing knowledge and information or promoting innovation (Jones et al., 2010) - see questions Q10 and 
Q11. Importantly, each of the different frameworks and characterisations of resilience present a viable way of assessing subjective disaster resilience. Part of the problem, however, is that there are so many different existing frameworks, many often tailored to specific contexts (Bahadur et al., 2015). Choosing from amongst them inevitably injects some degree of bias, requiring careful thought and transparency. Above all, any adoption of a multi-question approach would require considerable further research to validate they selection as an appropriate way of framing a set of resilience-related questions.

A further consideration is that any weighting of the different questions is likely to be subject to various assumptions and methodological weaknesses. Assigning weights can either be done though simplistic and naïve means (such as assuming that each question or category of questions is equally important) or more empirically (such as the use of various statistical analysis to decide on weighting of each question). Neither approach is perfect; and judgement calls are required in deciding which methods are best suited to the objectives of any research programme.

Perhaps the best way of ensuring accurate assessments of subjective resilience is for a number of different approaches to be trialled and tested. Above all, maintaining a diversity of methods and approaches that range in complexity, scope and focus will be important in gaining a more holistic understanding of resilience.

\section{Challenges to the assessment of subjective resilience and how to address them}

Subjective approaches to the measurement of socioeconomic characteristics such as poverty, well-being or resilience are each affected by a number of well-documented biases and methodological challenges. While many of them can be accounted for through thorough careful design of methods, they nonetheless require consideration.

A commonly cited concern is whether self-reported responses can be considered valid (Diener et al., 2002). After all, reports of subjective resilience do not reflect a stable state of household resilience. Rather, they are a judgement that individuals provide on the spot, based on information that is available to them at the time and influenced by myriad contextual and emotive factors (Schwarz \& Strack, 1999). Traits such as personality, values and beliefs may have a significant effect on how two people with the same levels of resilience self-report themselves. This is particularly the case with measures of subjective well-being, where scores can vary during the day depending on a number of endogenous and exogenous factors such as the weather, time of day, or where the question appears in relation to others (Strack et al., 1991).
Understanding how they affect scores is important, as we would not necessarily expect a household's resilience to disasters to fluctuate largely on a day-to-day basis without considerable forcing. Yet, these influencing factors do not present grounds for dismissing subjective measures altogether as 'the idiosyncratic effects of recent, irrelevant events are likely to average out over representative samples' (Kahneman \& Krueger, 2006: 7). Thus, with careful survey design, multiple surveys over time and adequate sampling methods, many of these biases can be reduced and largely accounted for.

More widely, people tend to compare themselves with those around them. Therefore, respondents exposed to one culture may report a different score than compared to others from another culture, irrespective of any differences in their overall resilience (e.g. perhaps one culture has a more optimist take on life than another). This makes cross-cultural comparison difficult. One method of trying to reduce this bias is to ask people to respond relative to a reference point, such as their neighbours or an average person in their village/town. However, the effects of crosscultural bias require careful consideration in interpreting higher-level data, such as comparison between different countries. One option is the use of anchoring vignettes (Hopkins \& King, 2010), which provides people with a hypothetical example (such as the characterisation of a person highly vulnerable to flooding) and asks them to provide a subjective rating. Given that cultural values do not tend to shift quickly, it may also be more insightful to focus on longitudinal analyses (tracking resilience-scores over time in the same place) than cross-comparisons between other cultures or locales (tracking the difference between one place and another at the same moment in time).

A bias that is particularly important to account for is tactical reporting. For example, in areas that receive considerable development or humanitarian assistance in meeting people's basic livelihood needs, it is possible that respondents may choose to respond in their own self-interest, i.e. claiming to be more vulnerable than they actually are in the hope of securing sustained or increased levels of assistance. The opposite may equally be true, whereby people do not want to be considered as having low levels of resilience - perhaps due to the social stigma attached with the label - and deliberately claim that their household has a higher level of resilience than in reality. This is where a thorough understanding of the context and political economy of the surveyed area can be of immense value. Clear and neutral wording can also be important. Above all, it showcases the need to consider subjective information not as a stand-alone but as a useful complement to objective forms of data collection. 


\section{How can subjective resilience be used to inform decision making and better targeting of resilience-building activities?}

The collection of information related to subjective resilience can have a number of important practical uses. For a start, it can offer a quick, efficient and cost effective tool for M\&E of resilience-building initiatives. The assessment of subjective resilience at various stages of project implementation - prior, during and subsequent - allows valuable insights to be gained on how and where activities have influenced people's perceived disaster resilience over time. Most importantly it allows for inferences to be made with regard to the effectiveness of resilience-building initiatives - an issue of considerable interest to international donors, multilateral development agencies, governments and NGOs given the current scale of investments. Any attribution would, however, have to carefully consider the type of assessment and design of survey delivery (such as the use of Randomised Control Trials) in making any such claims. While measurement of the impact of interventions on subjective resilience can never provide a complete account of objective resilience (an intervention can lead to a person feeling more resilient whilst unwittingly placing them at greater risk to an unforeseen or underprepared risk), it can complement other information in evaluating and attributing the impact of external interventions from a recipient and bottom-up perspective.

At a higher level, the same tools may feasibly be applied to the evaluation of national or international resiliencebuilding initiatives, although this has so far proven difficult. If international policy commitments such as the Sendai Framework Disaster Risk Reduction (SFDRR) or the Sustainable Development Goals (SDGs) are working effectively, then it is only reasonable to expect a marked difference in how resilient local people perceive themselves to be. Large national and regionally representative surveys, such as Afrobarmeter or the Gallup World Poll, that collect longitudinal data over a long period of time could provide a valuable channel for such efforts. Indeed, similar approaches have been proposed for the evaluation of national social and economic policies by collecting well-being and life evaluation data (Dolan \& Metcalfe, 2012; Diener, 2000). Such a tool may therefore allow a way of holding NGOs, businesses and governments to account through a bottomup method that captures the collective voice of beneficiaries and those most affected by disaster events.

Lastly, information on subjective resilience can allow us to gain a more holistic and bottom-up perspective on our understanding of resilience at household and other scales. It can help to elaborate the relationship between subjective assessments of a household's disaster resilience and psychological and cultural factors such as attitudes, emotions, personality traits, beliefs and norms (Kruger et al., 2015). In addition, a more comprehensive understanding of household resilience allows us to better identify what factors contribute to increased (and decreased) resilience. In turn, this can feed into improved targeting of resilience-building activities at all levels of governance. By comparing objective and subjective assessments, further research should be able to indicate whether people who rate themselves as highly resilient also score high on objective measures of resilience, and vice versa. Conversely, it is highly likely that there will be areas where objective and subjective assessments differ. Understanding the drivers (and biases) for such disparities could point to different interpretations of resilience on the ground, as well as the effectiveness of resilience-building activities, and may point to different policy options.

\section{Conclusion}

In this paper, we outline the rationale for assessing subjective disaster resilience at the household level. While it is clear that any approach to subjective assessment will face significant methodological and conceptual challenges, we show these to be far from insurmountable. Most importantly, measuring subjective resilience offers a valuable opportunity to capture the perspectives of those who know most about their own resilience and the factors that contribute to it: the people themselves. Moreover, this type of information has a number of unique practical applications, such as helping to improve our understanding of what works and doesn't with regards to resiliencebuilding activities; enhanced targeting of resilience-related programmes and resources; as well as providing a useful bottom-up tool for capturing the voice of beneficiaries and local communities.

Establishing the feasibility and methodological robustness of a subjective approach to measuring disaster resilience will inevitably take time. However, a tremendous amount of knowledge can already be drawn from current understandings of household disaster resilience, as well as insights gained through gathering subjective information in related fields, such as subjective well-being and psychological resilience. Care should nonetheless be taken in examining the merits and limitations of various different approaches to measuring subjective resilience. It is likely that a range of methods, surveying tools and applications will be required to satisfy the diversity of user needs and resources available.

Ultimately, the aim here is not to entirely replace traditional methods of resilience measurement. On the contrary, objective measures are a vital component of the measurement process. Rather, if shown to be effective, we argue that bottom-up subjective methods should be used alongside objective methods, helping to capture many of the components of resilience that are difficult to observe and allowing people's perspectives to be heard in a systematic manner. Getting the process right will be an important step forward in gaining a more holistic understanding of what it takes for a household to be resilient to disaster risk. 


\section{References}

Adger et al. (2013) Human resilience to climate change and disasters: Response from University of Exeter. Accessible at: https://royalsociety.org/ /media/policy/projects/resilience-climate-change/parts1-20.pdf

Adger, W. N. (2000) 'Social and ecological resilience: are they related?' Progress in Human Geography 24(3): 347-364.

Adger, W. N., Dessai, S., Goulden, M., Hulme, M., Lorenzoni, I., Nelson, D. R., \& Wreford, A. (2009) 'Are there social limits to adaptation to climate change?' Climatic change 93 (3-4): 335-354.

Aldunce, P., Beilin, R., Howden, M., \& Handmer, J. (2015) 'Resilience for disaster risk management in a changing climate: Practitioners' frames and practices.' Global Environmental Change 30: 1-11.

Alexander, D. E. (2013) 'Resilience and disaster risk reduction: an etymological journey.' Natural Hazards and Earth System Science 13(11): 2707-2716.

Bahadur, A., Wilkinson, E., Tanner, T. (2015) 'Resilience frameworks: a review.' London: Overseas Development Institute.

Béné, Christophe et al. 'Resilience: new utopia or new tyranny? Reflection about the potentials and limits of the concept of resilience in relation to vulnerability reduction programmes.' IDS Working Papers 405 (2012): 1-61.

Berkes, F. \& Ross, H. (2013) Community Resilience: Toward an. Integrated Approach, Society \& Natural Resources: An. International Journal, 26:1, 5-20,

Blennow, K, and Johannes, P. (2009) 'Climate change: Motivation for taking measure to adapt.' Global Environmental Change 19.1 (2009): 100-104.

Blennow, K. et al. (2012). ‘Climate change: believing and seeing implies adapting.' PloS one 7.11 (2012): e50182.

Bowling, A. (2005) 'Just one question: If one question works, why ask several?' Journal of Epidemiology and Community Health 59(5): 342-345.

Brooks, N., Anderson, S., Ayers, J., Burton, I., \& Tellam, I. (2011) Tracking adaptation and measuring development. IIED. London: UK.

Brown, K., \& Westaway, E. (2011) 'Agency, capacity, and resilience to environmental change: lessons from human development, well-being, and disasters'. Annual Review of Environment and Resources 36(1): 321.

Burnard, K., \& Bhamra, R. (2011) 'Organisational resilience: development of a conceptual framework for organisational responses'. International Journal of Production Research 49(18): 5581-5599.

Burton, C., \& Cutter, S. L. (2008) 'Levee failures and social vulnerability in the Sacramento-San Joaquin Delta area, California'. Natural Hazards Review 9(3): 136-149.

Carpenter S.R., Walker, B.H., Anderies, J.M., Abel, N. (2001) 'From metaphor to measurement: resilience of what to what?', Ecosystems 4:765-781.

Clayton, S. et al. (2015) 'Psychological research and global climate change', Nature Climate Change, 5, 640-646. DOI: 10.1038/NCLIMATE2622.

Constas, M., \& Barrett, C. (2013) 'Principles of resilience measurement for food insecurity: metrics, mechanisms, and implementation plans'. Expert Consultation on Resilience Measurement Related to Food Security. Rome: Food and Agricultural Organization and World Food Program.

Costanza, Robert et al. (2009) 'Beyond GDP: The need for new measures of progress'. The Pardee Papers. University of Boston. Boston: USA.

Cumming, G. S., Barnes, G., Perz, S., Schmink, M., Sieving, K. E., Southworth, J., \& Van Holt, T. (2005) 'An exploratory framework for the empirical measurement of resilience', Ecosystems, 8(8), 975-987.

Cutter, Susan L et al. (2008) 'A place-based model for understanding community resilience to natural disasters', Global Environmental Change 18(4): 598-606.

Deiner, E., Lucas, R. E., \& Oishi, S. (2002) 'Subjective well-being: The science of happiness and life satisfaction', Handbook of Positive Psychology, 63-73.

Deiner, E., Lucas, R. E., \& Oishi, S. (2002). Subjective well-being: The science of happiness and life satisfaction. Handbook of Positive Psychology, 63-73.

DFID. (2011) Defining Disaster Resilience: A DFID Approach Paper. London: Department for International Development.

DFID. (2014) Building resilience and adaptation to climate extremes and disasters programme. London: Department for International Development. (https://www.gov.uk/international-development-funding/ building-resilience-and-adaptation-to-climate-extremes-and-disasters-programme).

Diener, E. D., Emmons, R. A., Larsen, R. J., \& Griffin, S. (1985) 'The satisfaction with life scale', Journal of Personality Assessment 49(1): 71-75.

Diener, E., Lucas, R. E., \& Oishi, S. (2002) 'Subjective well-being'. Handbook of Positive Psychology, 63-73.

Diener, Ed. (2000) 'Subjective well-being: The science of happiness and a proposal for a national index', American Psychologist 55(1): 34.

Djalante, R., \& Thomalla, F. (2011) 'Community resilience to natural hazards and climate change impacts: a review of definitions and operational frameworks', Asian Journal of Environmental Disaster Management 3(3): 339-355. 
Dolan, P., \& Metcalfe, R. (2012) ' Measuring subjective wellbeing: Recommendations on measures for use by national governments', Journal of Social Policy 41(02): 409-427.

Eakin, H. C., \& Wehbe, M. B. (2009) 'Linking local vulnerability to system sustainability in a resilience framework: two cases from Latin America', Climatic Change 93(3-4): 355-377.

Eakin, H., Benessaiah, K., Barrera, J. F., Cruz-Bello, G. M., \& Morales, H. (2012) 'Livelihoods and landscapes at the threshold of change: disaster and resilience in a Chiapas coffee community', Regional Environmental Change 12(3): $475-488$.

Eid, Michael, Langeheine, Rolf and Diener, Ed. (2003) 'Comparing Typological Structures Across Cultures By Multigroup Latent Class Analysis A Primer', Journal of Cross-Cultural Psychology 34(2): 195-210.

Elasha, B. O., Elhassan, N. G., Ahmed, H., \& Zakieldin, S. (2005) 'Sustainable livelihood approach for assessing community resilience to climate change: case studies from Sudan'. Assessments of Impacts and Adaptations to Climate Change (AIACC) Working Paper 17. Accessible at: http://www.start.org/Projects/AIACC_Project/working_papers/ Working\%20Papers/AIACC_WP_No017.pdf

Engle, Nathan L. (2011) 'Adaptive capacity and its assessment', Global Environmental Change 21(2): 647-656.

Folke, C. (2006) 'Resilience: the emergence of a perspective for social-ecological systems analyses', Global Environmental Change 16(3): 253-267.

Folke, C., Carpenter, S., Elmqvist, T., Gunderson, L., Holling, C. S., \& Walker, B. (2002) 'Resilience and sustainable development: building adaptive capacity in a world of transformations', AMBIO: A Journal of the Human Environment 31(5): 437-440.

Fordyce, M. W. (1988). A review of research on the happiness measures: A sixty second index of happiness and mental health. Social Indicators Research, 20(4), 355-381.

Fox, R. et al. (2012) The Characteristics of Resilience Building: A Discussion Paper. London: The Inter-agency Resilience Working Group.

Frankenberger, T. R., Constas, M. A., Nelson, S., \& Starr, L. (2014) 'How NGOs approach resilience programming', Resilience for Food and Nutrition Security 177.

Frey, Bruno S, and Stutzer, Alois. (2002) 'What can economists learn from happiness research?', Journal of Economic Literature (2002):402-435.

Gasper, D. (2005) 'Subjective and objective well-being in relation to economic inputs: puzzles and responses', Review of Social Economy 63(2): 177-206.

Graber, R., Pichon, F. and Carabine, E. (2015) Psychological Resilience: Capturing the State of the Research. Literature Review. London: Overseas Development Institute.

Grothmann Torsten and Patt, Anthony. (2005) 'Adaptive capacity and human cognition: The process of individual adaptation to climate change', Global Environmental Change 15(3):199-213.

GRP. (2014) Global Resilience Partnership, convened by Rockefeller and USAID http://www. globalresiliencepartnership.org/approach/

Hays, R. D., Sherbourne, C. D., \& Mazel, R. M. (1993) 'The rand 36冈item health survey 1.0', Health Economics 2(3): $217-227$.

Holling, C. S. (1973) 'Resilience and stability of ecological systems', Annual Review of Ecology and Systematics 1-23.

Hopkins, D. J., \& King, G. (2010) 'Improving anchoring vignettes designing surveys to correct interpersonal incomparability', Public Opinion Quarterly, nfq011.

IFRC. (2014) World Disasters Report: Focus on Culture and Risk. Geneva: The International Federation of Red Cross and Red Crescent Societies.

Jones, L., \& Boyd, E. (2011) 'Exploring social barriers to adaptation: insights from Western Nepal', Global Environmental Change 21(4): 1262-1274.

Kahneman, D., \& Krueger, A. B. (2006) 'Developments in the measurement of subjective well-being', The Journal of Economic Perspectives 20(1): 3-24.

Keating, A., Campbell, K., Mechler, R., Michel邓Kerjan, E., Mochizuki, J., Kunreuther, H., Bayer, J., Hanger, S., McCallum, I., See, L., Williges, K., Atreya, A., Botzen, W., Collier, B., Czajkowski, J., Hochrainer, S., Egan, C. (2014) Operationalizing Resilience Against Natural Disaster Risk: Opportunities, Barriers and A Way Forward. Switzerland: Zurich Flood Resilience Alliance.

Krueger, A. B., \& Schkade, D. A. (2008) 'The reliability of subjective well-being measures', Journal of Public Economics 92(8): 1833-1845.

Krüger, F., Bankoff, G., Cannon, T. and Schipper, L. (2015) Cultures and Disasters: Understanding Cultural Framings in Disaster Risk Reduction. Abingdon, UK: Routledge.

Kuruppu, N., \& Liverman, D. (2011). Mental preparation for climate adaptation: The role of cognition and culture in enhancing adaptive capacity of water management in Kiribati. Global Environmental Change, 21(2), 657-669.

Lee, T.R., (1986) 'Effective Communication of Information about Chemical Hazards', The Science of the Total Environment (51): 149-183. 
Levine, S. (2014) Assessing resilience: why quantification misses the point. London: Overseas Development Institute.

Lindell Michael K. and Perry, Ronald W. (2004) Communicating Environmental Risk in Multiethnic Communities. London: Sage.

Lockwood, M., Raymond, C. M., Oczkowski, E., \& Morrison, M. (2015) 'Measuring the dimensions of adaptive

capacity: a psychometric approach', Ecology and Society 20(1): 37.

Manyena, S. B. (2006) 'The concept of resilience revisited', Disasters 30(4): 434-450.

Marshall, N. A. (2010) 'Understanding social resilience to climate variability in primary enterprises and industries', Global Environmental Change 20(1): 36-43.

Marshall, N. A., \& Marshall, P. A. (2007) 'Conceptualizing and operationalizing social resilience within commercial fisheries in northern Australia', Ecology and Society 12(1): 1.

McGillivray, Mark and Clarke, Matthew (2006) 'Human Well-being: Concepts and Measures', In Mark McGillivray and Matthew Clarke, (eds.) Understanding Human Well-Being. Basingstoke: Palgrave MacMillan.

McIvor, D., \& Paton, D. (2007) 'Preparing for natural hazards: normative and attitudinal influences', Disaster Prevention and Management: An International Journal 16(1): 79-88.

Miller, F., Osbahr, H., Boyd, E., Thomalla, F., Bharwani, S., Ziervogel, G., \& Nelson, D. (2010) 'Resilience and vulnerability: complementary or conflicting concepts', Ecology and Society 15(3): 11.

NEF. (2012) Measuring Well-being: A guide for practitioners. London: New Economics Foundation.

Nelson, D. R., Adger, W. N., \& Brown, K. (2007) 'Adaptation to environmental change: contributions of a resilience framework', Annual Review of Environment and Resources 32(1): 395.

Nguyen, K. V., \& James, H. J. (2013) 'Measuring household resilience to floods: A case study in the Vietnamese Mekong river delta', Ecology and Society 18(3): 13.

O'Brien, K. 2009. Do values subjectively define the limits to climate change adaptation? In Adapting to climate change: Thresholds, values, governance, ed. W.N. Adger, I. Lorenzoni, and K. O’Brien, 164-180. Cambridge, UK: Cambridge University Press.

Oddsdóttir, F., Lucas, B., \& Combaz, É. (2013) ‘Measuring disaster resilience.' GSDRC Helpdesk Research Report 104. UK: GSDRC, University of Birmingham.

Odum, E. P. (1985) 'Trends expected in stressed ecosystems', Bioscience 35: 419-422.

OECD. (2013) 'OECD Guidelines on Measuring Subjective Well-being'. OECD Publishing.

Pasteur, K. (2011) From Vulnerability to Resilience: A framework for analysis and action to build community resilience. Bourton-on-Dunsmore: Practical Action Publishing.

Pelling, M. (2003) The vulnerability of cities: natural disasters and social resilience. Earthscan, London.

Guillen-Royo, M., \& Velazco, J. (2006). Exploring the relationship between happiness, objective and subjective wellbeing: Evidence from rural Thailand. WeD Working Paper 16. Bath: Wellbeing in developing countries WeD Research Group, University of Bath.

Scali, J., Gandubert, C., Ritchie, K., Soulier, M., Ancelin, M. L., \& Chaudieu, I. (2012) 'Measuring resilience in adult women using the 10-items Connor-Davidson Resilience Scale (CD-RISC). Role of trauma exposure and anxiety disorders', PloS one,7(6).

Schwarz, N., \& Strack, F. (1999) 'Reports of subjective well-being: Judgmental processes and their methodological implications', In: Daniel Kahneman, Ed Diener and Norbert Schwarz (eds) Well-Being: The Foundations of Hedonic Psychology. New York: Russell Sage Foundation: 61-84.

Scoones, I. (1998) Sustainable rural livelihoods: a framework for analysis. Brighton: IDS.

Seara, T. (2014). "Impacts of management on fisheries diversity as a factor affecting social resilience in communities of the southern new england region”. Open Access Dissertations. Paper 137. http://digitalcommons.uri.edu/oa_diss/137

Sen, A. (1985) Commodities and Capabilities, Oxford: Oxford University Press.

Singh, Kamlesh, and Yu, Xiao-nan. (2010) 'Psychometric evaluation of the Connor-Davidson Resilience Scale (CD-RISC) in a sample of Indian students', Journal of Psychology 1(1): 23-30.

Speranza, C. I., Wiesmann, U., \& Rist, S. (2014) 'An indicator framework for assessing livelihood resilience in the context of social-ecological dynamics', Global Environmental Change 28: 109-119.

Stevenson, B., Wolfers, J. (2008) Economic Growth and Subjective Well-Being: Reassessing the Easterlin Paradox, Brookings Papers on Economic Activity, Economic Studies Program, The Brookings Institution, vol. 39(1 (Spring), pages 1-102

Strack, F. E., Argyle, M. E., \& Schwarz, N. E. (1991) Subjective well-being: An interdisciplinary perspective. Oxford: Pergamon Press.

Suh, E., Diener, E., Oishi, S., \& Triandis, H. C. (1998) 'The shifting basis of life satisfaction judgments across cultures: Emotions versus norms', Journal of Personality and Social Psychology 74(2): 482.

Tanner, T.M. et al. (2015) 'Livelihood resilience in the face of climate change', Nature Climate Change 5: 23-26.

Twigg, J. (2009), Characteristics of a disaster-resilient community: a guidance note (version 2). London: University College London. Ungar, M. (2011) 'The social ecology of resilience: Addressing contextual and cultural ambiguity of a nascent construct', American Journal of Orthopsychiatry 81(1): 1-17. 
Urry, H. L., Nitschke, J. B., Dolski, I., Jackson, D. C., Dalton, K. M., Mueller, C. J., \& Davidson, R. J. (2004) ‘Making a life worth living neural correlates of well-being', Psychological Science 15(6): 367-372.

USAID. (2009) Community Resilience: Conceptual Framework and Measurement Feed the Future Learning Agenda. United States Agency for International Development. Rockville, MD: Westat.

Uy, Noralene, Takeuchi, Yukiko and Shaw, Rajib. (2011) 'Local adaptation for livelihood resilience in Albay, Philippines', Environmental Hazards 10(2): 139-153.

Varelius, J. 'Objective explanations of individual well-being', Journal of Happiness Studies 5(1): 73-91.

Veenhoven, R. (1990) 'Inequality in happiness: inequality in countries compared across countries'. Paper presented at the 12th World Congress of Sociology, Madrid, Spain.

Veenhoven, Ruut. (2012) 'Cross-national differences in happiness: Cultural measurement bias or effect of culture' International Journal of Wellbeing 2(4).

Walker, B.H., Ludwig, D., Holling, C.S. and Peterman, R.M., (1969) 'Stability of Semi-Arid Savanna Grazing Systems', Journal of Ecology 69 (2): 473-498.

Windle, Gill, Bennett, Kate M. and Noyes, Jane. 'A methodological review of resilience measurement scales', Health and Quality of Life Outcomes 9(8): 1-18.

Wu, G., Feder, A., Cohen, H., Kim, J. J., Calderon, S., Charney, D. S., \& Mathé, A. A. (2013) 'Understanding resilience’, Frontiers in Behavioral Neuroscience 7(10): doi:10.3389/fnbeh.2013.00010. 
ODI is the UK's leading independent think tank on international development and humanitarian issues.

Readers are encouraged to reproduce material from ODI Reports for their own publications, as long as they are not being sold commercially. As copyright holder, ODI requests due acknowledgement and a copy of the publication. For online use, we ask readers to link to the original resource on the ODI website. The views presented in this paper are those of the author(s) and do not necessarily represent the views of ODI.

(C) Overseas Development Institute 2015. This work is licensed under a Creative Commons AttributionNonCommercial Licence (CC BY-NC 3.0).

ISSN: 2052-7209

All ODI Reports are available from www.odi.org

Cover photo: CGIAR Research Programme on Dryland Systems: Indigenous knowledge for resilience in marginal drylands - Tunisia

\section{www.odi.org}

